\title{
A comparison of inexpensive statistical packages for microcomputers running MS-DOS
}

\author{
DARRELL L. BUTLER and WILLIAM NEUDECKER \\ Ball State University, Muncie, Indiana
}

\begin{abstract}
The purpose of the present paper is to describe and compare some inexpensive software packages that calculate a variety of statistics on microcomputers running MS-DOS. For each package, hardware requirements, program capabilities, limitations, accuracy, error handling, and other features are considered.
\end{abstract}

In recent years, there have been valuable comparative reviews of statistical software for MacIntosh (Lehman, 1986) and Apple II (Butler \& Jones, 1987) computers, as well as of very high-powered packages for the IBM PC (Fridlund, 1988; "Statistical Analysis Software,"' 1988). Fridlund compared eight packages and found Systat/ Sysgraph (\$795) and SPSS/PC Plus (\$795 for the base package) to be best. The broad review carried in PC Week ("Statistical Analysis Software") did not make evaluative comparisons.

Many users may be interested in finding relatively comprehensive, inexpensive MS-DOS packages for use in teaching and research laboratories. The descriptions provided here, of statistical packages costing $\$ 100$ or less, will hopefully be valuable in and of themselves and will provide useful standards for evaluating new packages that become available.

A variety of sources were used to find packages, including previous reviews (e.g., Butler \& Eamon, 1985), advertisements in computer and statistical journals and magazines, and listings of programs. Packages were selected if they calculated a reasonable variety of statistical procedures (i.e., not just ANOVA or regression), cost $\$ 100$ or less, and could run on MS-DOS machines. Ten packages were obtained: Exploring Statistics, ISP, KeyStat, Kwikstat, Minitab for students, Number Cruncher, PBStatistics, SPSS PC Studentware, Statistical Software for Microcomputers, and Superstat (See Appendix for vendors). Two other packages, EcStatic and Psych-Stats, were found, but user tests could not be completed. Information on EcStatic, taken from advertisements, is included in the summaries below. Detailed information on PsychStats could not be obtained. The addresses of the suppliers of these packages are also given in the Appendix. Some readers may wish to note that many of these packages were designed specifically for educational use. Note especially Exploring Statistics with the IBM PC and ISP, both of which contain routines other than statistical calculations.

Requests for reprints should be sent to Darrell L. Butler at the Department of Psychological Science, Ball State University, Muncie, IN 47306.

\section{GENERAL CHARACTERISTICS OF PACKAGES}

Table 1 summarizes the main general features, elementary statistical capabilities, and several other features of the packages. Although most of the entries in the table should be easy to understand, they are briefly described below. Characteristics difficult to describe in a table or beyond those listed in Table 1 are presented for each individual package following the description of the features that the table includes.

\section{General Features}

The packages varied substantially in their minimum memory requirement, from $64 \mathrm{~K}$ to $640 \mathrm{~K}$ RAM. Some of the packages can run on an inexpensive single disk drive machine, whereas some require two disk drives. All of the packages came with documentation, but there was great variability in its amount (see Table 1) and quality. One of the most important dimensions of quality was whether or not examples were included. Some diskettes containing packages could not be copied; others were not copy protected. Copy-protected programs are a major problem for users with hard-disk systems. All of the packages were fairly easy to get started, in part because most were menu driven. However, user friendliness varied substantially. For example, error trapping (that is, a program's ability to detect unacceptable keypresses or division by zero and provide guidance to the user) varied from poor to excellent. One surprising finding was that not only were these packages already inexpensive, many of their suppliers were willing to provide educational discounts.

\section{Statistics}

As in Butler and Jones (1987), the statistics calculated by the programs were compared to a list of common elementary statistical routines. The statistics shown in Table 1 were the ones described in at least half of 20 introductory statistics textbooks. For each statistical routine, the program's limitations were studied: maximum number of scores, maximum number of groups or cells (where 


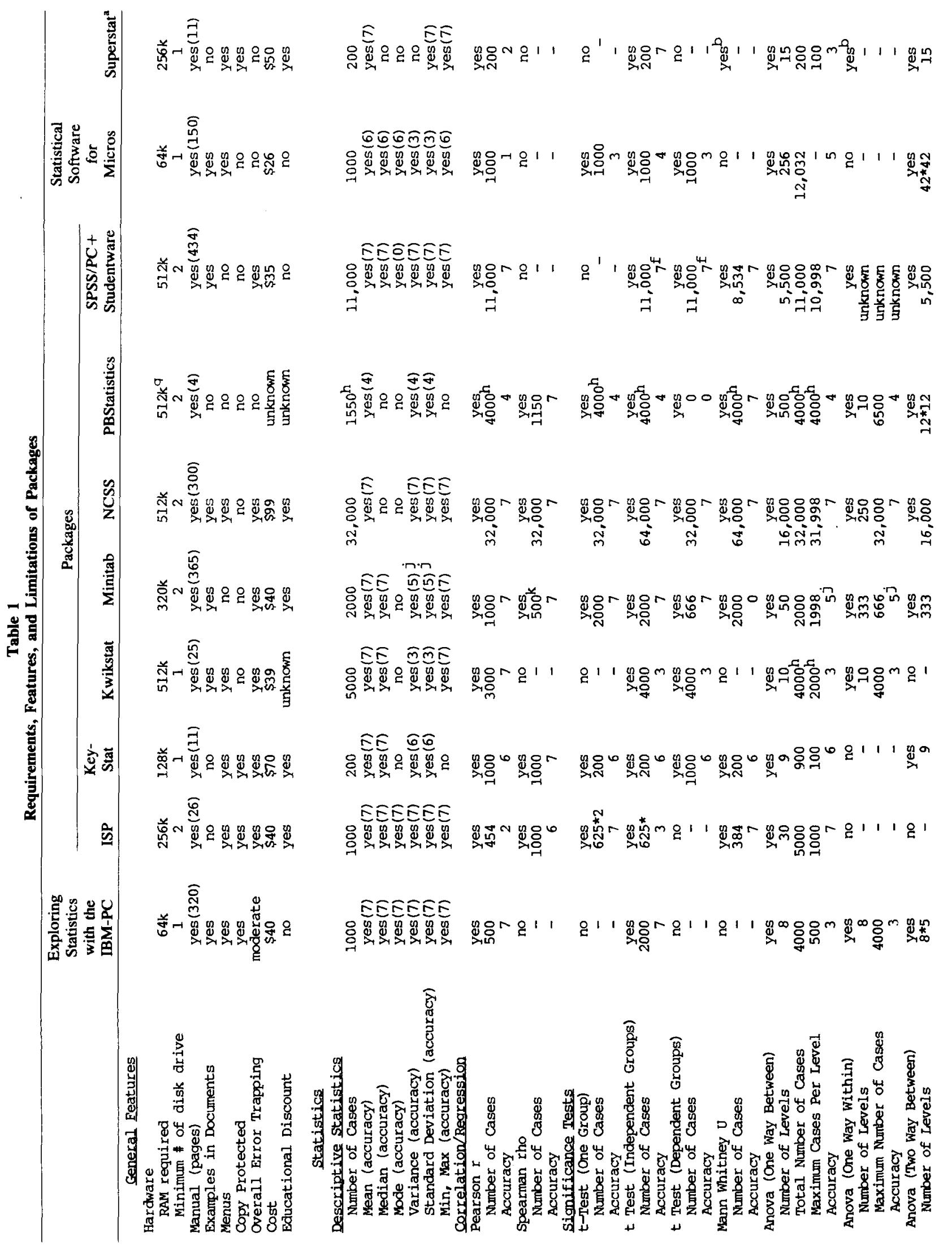







appropriate), and accuracy. Accuracy was studied as in Butler and Eamon (1985) and Butler and Jones (1987). To test accuracy, small data sets that varied in the number of significant digits (with known mean, variance, etc.) were run through all of the routines. Researchers interested in analyzing dates (e.g., 1988) or msec reaction times (e.g., 2,594) would be interested in packages capable of handling data with at least four digits of accuracy. Since small data sets are used here, our tests are probably conservative. Table 1 indicates the maximum number of digits that one could use in a data set and obtain correct statistical output. The maximum studied here was seven digits, because psychological data rarely would go beyond this limit. No benchmarks for speed were run.

\section{Other Features}

One surprising finding was that all but one of these inexpensive programs contained at least some data graphics and the graphics capabilities were good.

Most of the packages used spreadsheet formats for data entry and editing, although a few utilized less user-friendly techniques. Error trapping during data entry and editing also varied substantially, from poor to excellent. Some of the packages contained data-generation proceduresthat is, the user could generate data with a uniform distribution or some other distribution. This is a useful characteristic for packages employed in instruction. Some of the packages permitted a variety of data transformations (e.g., logarithms) and permitted users to save data on disks. The packages differed substantially in data structures (i.e., the form of data stored on the disk) and thus in their ability to use data saved by other programs. They also differed in their abilities to save predicted or residual scores for further analysis or plotting.

\section{UNIQUE PROGRAM FEATURES}

Features not described in Table 1 are described below for each individual package. Two users (one student and one professor) examined the packages in detail.

\section{EcStatic}

(This package was not obtained in time for review. The comments included here are based upon advertisements.) The package requires 640K RAM. The cost is $\$ 49.95$, but quantity and educational discounts are available. The accompanying user guide has an index. The program is easy to use, in part because it is menu driven.

Up to 255 variables can be defined, and the number of cases is dependent only on disk space. A full screen data editor is included in the package. Among the statistics available are the following: descriptive statistics, Pearson correlation, Spearman \& Kendall correlation, $t$ tests, Wilcoxon, ANOVA (including repeated measures and covariance analysis), Kruskal-Wallis, chi-square contingency, and multiple regression. The package can create scatter plots, frequency distributions, and histograms. Data transformations are available.

\section{Exploring Statistics with the IBM PC}

This package is designed for use in statistics courses. It comes with a well-written manual that serves as a type of statistics workbook. It includes numerous examples and exercises for the student.

The package provides many calculations besides those summarized in Table 1. First, some routines (correlation and chi-square) include many intermediate calculations in output. Second, the package calculates a number of statistics not described in Table 1: quartiles, 3rd and 4th moments, skewness, kurtosis, geometric means, average deviation, coefficient of variation, binomial test, multiple linear regression (a maximum of 16 variables and 400 cases), and time series analysis. Third, the package includes many routines that could be helpful in classrooms or to individuals learning statistics. For example, one routine creates random numbers from various distributions (normal, poisson, and uniform). Another routine is a Monte Carlo demonstration of the central limit theorem. A third routine demonstrates the law of large numbers. In addition, there are some other potentially useful routines in this package. There is a routine for creating box plots. The MATCOR program reads several data files and then prints a compact matrix of correlation coefficients among the files. There are also routines for splitting, transferring, and sorting data files.

From the student's point of view, this package can be very useful, but it has some significant problems. Some calculations are not error-trapped well. For example, a perfect correlation leads to a divide by zero error. The return key is needed after some responses, but not others. As a user begins to get familiar with a routine, errors may be caused because the return key is pressed when it shouldn't be.

From a professional's viewpoint, this package could be of use in teaching elementary statistics or an introduction to methodology, on the undergraduate level. However, some important routines are missing. Although the index includes a related-samples $t$ test, the reference could not be found in the package. Simple statistics such as $z$ and $t$ tests should be available for elementary courses. However, this package may not be appropriate for more advanced statistics courses. The ANOVA is limited to two independent variables.

\section{Key-Stat}

The correlation/regression program is much more complete than most packages. Output of the regression routine includes descriptive statistics and many values used in calculating the correlation coefficient. A scatter plot as well as a regression equation are generated. The bestfitting line can be included in the scatter plot. Also, in addition to the statistics listed in Table 1, Key-Stat has a multiple regression routine (up to 8 predictors and 100 cases) and a rather elaborate calculator that simulates a Hewlett-Packard RPN calculator.

From the student's viewpoint, this package was one of the easiest to use of those reviewed here. A unique aid 
that this package provides the novice is a program that questions the user to help locate the appropriate test statistic. However, some of the menus (especially the opening menu) use terminology that makes it somewhat difficult to find particular statistical routines.

From the professional's viewpoint, this package does not have enough power to be useful for most research projects or advanced laboratory courses. It performs no statistical calculations other than those described in Table 1 and the few described above. However, it also does not really contain any instruction in statistics. It can be a useful simple statistical calculator. It may be useful for students (especially undergraduates) in elementary statistics courses or courses in introductory laboratory methods.

This package was originally available for Apple II computers and has been converted to run on MS-DOS machines. The program is very similar to the Apple II version (see Butler \& Jones, 1987). The major differences between the Apple II and the present version are that the MS-DOS version costs $\$ 20$ more, has greater consistency among routines, and is slightly less accurate according to the tests run here. For another review, see Henry and Bauer (1986). Version 2.0 (being beta tested at the end of 1988) has better accuracy, compatibility with DIF files, and other improvements.

\section{NCSS}

This package is powerful and reasonably complete. In addition to the statistics shown in Table 1, NCSS computes ANOVAs with up to 10 independent variables (but only a few hundred degrees of freedom in the entire analysis), a variety of post hoc tests (e.g., Newman-Keuls, Fisher, LSD, and Duncan's), forecasting by five different methods, multiple regression with over 200 predictors, discriminant analysis, factor analysis, cluster analysis, several nonparametric tests (e.g., Wilcoxon, Wald-Wolfowitz runs test, sign test, and Fisher's exact test), and more.

The manuals are needed for one to be able to use this package. Fortunately, the two manuals (one on statistics and one on graphics) are very well done. They include descriptions of the variety of options and examples of how to execute most of them.

This is an excellent package for the professional. It is accurate, it handles reasonably large files, and it is very complete. It could be used in some laboratories and in many graduate statistics courses. It probably has too much power to be useful in most undergraduate statistics courses. One weakness is the spreadsheet editor, which is slow for large data files. Another weakness is that the program cannot execute ANOVAs on within-subject experiments with many degrees of freedom. From the student's perspective, the package is a little overwhelming, particularly for novices. Because it has so many options, a novice would need guidance.

\section{ISP}

This package is a very user-friendly system for the teaching of statistics. In addition to the statistics shown in Table 1, this package includes six different forecasting procedures, the Wilcoxon test, and the coefficient of concordance. Seven distributions are accessible as tables of probabilities, probability of a particular statistical value, or cumulative probabilities. A new version (V 3.0), with some new routines (e.g., two-way ANOVAs) and enhanced graphics, is now available. Besides calculation routines, this package includes self-teaching modules, exercises, and simulations for use in classroom demonstrations, all of which provide more than just the necessary information. For example, assumptions of tests and some intermediate values in calculations are presented.

The package does have some problems. The MannWhitney $U$ procedure uses an approximation formula. As a caution, a minimum sample size of 30 is required by the package for this routine. I talked with one of the package authors, Robert Winkler, and Mike Harde, a representative of Lincoln Systems. They expressed a genuine interest in feedback from users and a desire to improve the program in directions users request.

From the student's perspective, this is a very good program, the best instructional package among those we examined. It helps the student learn and calculate statistics in a very user-friendly environment.

From a professional viewpoint, this package could be useful in undergraduate courses and perhaps some graduate courses. Because the package authors are in a business school, they emphasized trend analysis over ANOVA. This could cause problems for some psychology instructors.

\section{Kwikstat}

This package was designed for students and occasional users of a statistics package. There is a well-done on-line help system in addition to the manual. The tutorial helps with all the routines described in Table 1 as well as the few others that are calculated: multiple regression (10 predictors; 300 cases), Newman-Keuls, and 3-D crosstabs. Also, some extra graphics routines are included, box plots and 3-D box charts.

We had a few problems with this package. For example, the correlation routine cannot calculate a perfect correlation. This package is probably too limited to be of much value in instruction or research.

\section{Minitab for Students}

Minitab contains most common elementary statistical routines. In addition to those summarized in Table 1 , this package calculates $z$ tests, confidence intervals, sign test, Wilcoxon, multiple regression, and time series analyses. It also creates several graphs other than those reported in Table 1, such as box plots and stem and leaf displays. Tutorials are provided for all the commands. 
The data is placed on a spreadsheet. Simple commands make it possible to execute most statistics one step at a time. However, there are commands to run most statistics quickly and efficiently. One exception is the chisquare goodness of fit, for which there is no command. Rather, the manual shows how to calculate the statistic step by step, using the spreadsheet commands. One interesting characteristic of this package is the capacity to create and store MACROs-that is, sequences of commands. Theoretically, MACROs could be created for statistics not presently available in the package.

This package has some problems. Many output values are rounded. As a result, the data graphs often look unusual, and post hoc tests should be cautiously performed on the data in ANOVA summary tables. The greatest problem concerned the Mann-Whitney $U$ test. When the data contained more than three digits, the computer "hung" and had to be rebooted.

From the student's point of view, Minitab was not the most user-friendly program reviewed here. However, it was easier to use than the SPSS student version. With the manual, which is easy to follow, and a little introduction, most routines can be executed fairly easily. This package could be very useful to students in statistics courses.

From the professional's viewpoint, this is a very good instructional package. Because of the substantial accuracy and flexibility in most of the commands and MACROs, it could be used in most if not all statistics classes, especially on the advanced undergraduate or the graduate level. The most significant problem is its weaknesses with respect to within-subject ANOVAs. I recommend the professional version of Minitab, not the student version, for complex or within-subject ANOVAs.

\section{PBStatistics}

This is a collection of 60 individual programs. Three of the programs are editors, about ten concern distributions, and the remainder are statistical routines. In addition to the routines reported in Table 1, the package calculates the Kolmogorov-Smirnov test, Wald-Wolfowitz runs test, the Wilcoxon test, Kendall concordance, ANOVAs (up to three-way), ANOVA (up to three-way), multiple regression (median test), a variety of post hoc tests for ANOVA, point-biserial correlation, Kendall fair correlation, Kolmogorov single sample test, sign test, Bartlett homogeneity of variances, Cochran test, Fisher's exact test, Hartley F-Max, McNemar test, and Walsh test.

In many respects, this package is unfinished. Students have problems with the limited documentation, lack of error trapping, and lack of menus. In addition, it has no graphics, and the editors cause confusion. Each editor creates a slightly different data structure. One is for betweengroup analyses, one for dependent-group analyses, and one for mixed-group analyses. Also, once one requests a printer listing, it cannot be turned off. On the positive side, each program is fairly simple to run.
From the professional's viewpoint, this package has significant strengths and weaknesses. One impressive characteristic is the number of nonparametric routines (18). On the negative side, the lack of accuracy of parametric routines makes the package less useful in teaching and research. The package may be useful in some laboratories and in courses concerning nonparametrics.

\section{SPSS/PC + Studentware}

In addition to the statistics summarized in Table 1, this package calculates ANOVAs with up to five independent variables and the sign test. However, its files and commands are compatible with the more expensive SPSS/PC+ and the mainframe versions of SPSS, so they could be used if the limits of this program were reached. The version of SPSS reviewed here is designed for use in statistics courses, as is obvious from the name and the 210 page instructor manual that accompanies the package. For a number of reasons, including the fact that the package uses a command language instead of menus, this package is harder to learn to use than the other packages described in the present review.

From the student's point of view, this package is hard to use without some guidance. However, it does come with an adequate manual, on-screen help, and good error checking! It comes on 5 diskettes $\left(5^{1 / 4} 4^{\prime \prime}\right)$, which means that a lot of disk swapping is required. Furthermore, the commands require precise syntax (for example, one can't forget periods at the end of command lines).

From the professor's viewpoint, this package could be useful in the teaching of elementary statistics. It has the advantage of vertical integration: students can easily move to the more complete and complex versions of SPSS as they progress through the undergraduate and graduate curriculum. However, it will still take more time to instruct students in how to use this program than it will with others. Both the instructor's manual and the student manual contain 25 chapters each. It is unclear how many lectures would be needed to communicate the information on SPSS contained in these chapters.

\section{Statistical Software for Micros}

In addition to the statistics summarized in Table 1, this package calculates a variety of discrete trial probabilities, including the binomial and normal approximation to the binomial.

From the student's and the professional's perspective, this was the package with the most weaknesses. First, it is the slowest of those reviewed here. It is apparently not written in compiled code, and it is not very well-optimized for speed. Second, error trapping is very poor. In fact, for many types of errors, the computer just "hangs." This can only be discovered by pressing keys and finding that no interaction is possible. However, some calculations we ran were so slow (over $20 \mathrm{~min}$ ) that we had to be careful 
about pressing keys or we would disrupt calculations and have to start over. For most kinds of errors, the MS-DOS disk had to be rebooted. Third, data files are not compatible across routines. Data had to be reentered for each type of test run. Fourth, some minor programming errors cause difficulties. For example, in the two-way ANOVA routine, the cap locks must be on or the package will only accept data from a disk file.

\section{Superstat}

This package is the least sophisticated of those reviewed. The data editor is a separate program that must be run independently of the statistics program. Unfortunately, it is not a sophisticated editor. The company that distributes Superstat recommends using a spreadsheet to enter and edit data. However, there are some terminology problems that make it difficult to do this. The words observations and variables are used ambiguously in Superstat. This created great difficulty for setting up a data file for a two-factor ANOVA, as well as for other routines. In addition, there is no manual that explains how to set up data files for the various routines.

From the student's perspective, this program is unacceptable. It has no error trapping. Once an error is made, the user cannot back up or recover. The only option is to reboot the system (using a different diskette) and start over. On many occasions, the package was very confusing. The on-line help messages often didn't help, and the manual was designed only to be used as a technical reference source. Many routines could not be run. For example, documentation claimed that the package could compute the Mann-Whitney $U$ test and the Kruskal-Wallis test, but they could not be found.

From the professional's viewpoint, this package is too weak and inefficient to be of much value. Only individuals with substantial statistical knowledge and patience can use it to execute a within-subjects ANOVA. Some additional power is obtainable if a spreadsheet program is used to enter data. Then the number of cases will be much higher than what is shown in Table 1 (according to documentation, the only limit is disk space). However, since the accuracy was low for ANOVAs, this extra effort is not justifiable.

\section{CONCLUSION}

The majority of the inexpensive statistics packages for MS-DOS machines are targeted for students. Those that impressed us most for instruction were ISP, Minitab, and for some users, PBStatistics. For research, the only package with reasonable power was NCSS. In fact, NCSS may provide "the most bang for the buck" among packages available today.

\section{REFERENCES}

Butler, D. L., \& EAmon, D. B. (1985). An evaluation of statistical software for research and instruction. Behavior Research Methods, Instruments, \& Computers, 17, 352-358.
Butler, D. L., \& Jones, S. K. (1987). A comparison of inexpensive statistical packages for Apple II microcomputers. Behavior Research Methods, Instruments, \& Computers, 19, 99-103.

Fridlund, A. J. (1988, September 19). Statistics software. Infoworld, pp. 55-76.

Henry, N. W., \& Bauer, D. F. (1986). Key-Stat. American Statistician, 40, 50-51.

Lehman, R. S. (1986). MacIntosh statistical packages. Behavior Research Methods, Instruments, \& Computers, 18, 177-187.

StatistiCal aNALYSIS SOFTWARE (1988, October 24). PC Week, 5(43), $116-121$

\section{APPENDIX}

\section{Program Suppliers' Addresses}

EcStatic

SomeWare in Vermont, Inc.

P.O. Box 215

Montpelier, VT 05602

(800) $451-4580$ or (802) 229-4974

Exploring Statistics with the IBM PC V 2.0

Addison-Wesley Publishing Company, Inc.

Attn: Order Department

Reading, MA 01867

(617) 944-3700

ISP [i.e., Interactive Statistical Programs]

Lincoln Systems Corporation

P.O. Box 391

Westford, MA 01886

(508) 692-3910

Key-Stat

Oakleaf Systems

P.O. Box 472

Decorah, IA 52101

(319) $382-4320$

Kwikstat

Mission Technologies

P.O. Box 1169

Cedar Hill, TX 75104

(214) 291-2115

NCSS V 5.1 [i.e., Number Cruncher Statistical System] c/o Dr. Jerry L. Hintze

865 East 400 North

Kaysville, UT 84037

(801) 546-0445

\section{PBStatistics}

Public Brand Software

P.O. Box 51315

Indianapolis, IN 46251

(800) IBM-DISK

or (317) 856-4144

or (317) 856-1001

(or the author can be contacted directly:

John V. Flowers

Chapman College

Dept. of Psychology

333 N. Glassell Ave.

Orange, CA 92666) 
SPSS/PC+ Studentware

SPSS Inc.

444 N. Michigan Avenue

Chicago, IL 60611

(312) $329-3600$

Psych-Stats

National Collegiate Software Clearinghouse

NCSU Box 8101

Raleigh, NC 27695-8101
Statistical Software for Micros

Kern International, Inc.

433 Washington St.

P.O. Box 1029CA

Duxbury, MA 02331

(617) 934-0445

Superstat V 3.0

Serendipity Systems

P.O. Box 3293

Valdosta, GA 31604

(no phone number available) 